\title{
Carbohydrate Binding Properties of the Stinging Nettle (Urtica dioica) Rhizome Lectin ${ }^{1}$
}

\author{
NAOTO SHIBUYA, IRWIN J. GOLDSTEIN, ${ }^{2}$ JULES A. SHAFER, \\ WILLEY J. PEUMANS, ${ }^{*}$ AND WILLEM F. BROEKAERT*
}

\author{
Department of Biological Chemistry, The University of Michigan, Ann Arbor, Michigan 48109, and \\ *Laboratorium voor Plantenbiochemie, KU Leuven, Kardinaal Mercierlaan, 92, B-3030 Leuven, Belgium
}

Received March 3, 1986

The interaction of the stinging nettle rhizome lectin (UDA) with carbohydrates was studied by using the techniques of quantitative precipitation, hapten inhibition, equilibrium dialysis, and uv difference spectroscopy. The Carbohydrate binding site of UDA was determined to be complementary to an $N, N^{\prime}, N^{\prime \prime}$-triacetylchitotriose unit and proposed to consist of three subsites, each of which has a slightly different binding specificity. UDA also has a hydrophobic interacting region adjacent to the carbohydrate binding site. Equilibrium dialysis and uv difference spectroscopy revealed that UDA has two carbohydrate binding sites per molecule consisting of a single polypeptide chain. These binding sites either have intrinsically different affinities for ligand molecules, or they may display negative cooperativity toward ligand binding. (c) 1986 Academic Press, Inc.

Plant lectins are a group of (glyco)proteins with the ability to recognize and bind specific sugar residues $(1,2)$. Although most well-characterized plant lectins have been isolated from seeds, especially those of legumes, the number of plant lectins isolated from the other parts of the plant has become of increasing interest especially as it may relate to the metabolism and physiological role of these carbohydrate-binding proteins. For example, lectins were isolated and characterized from such tissues as the leaves $(3,4)$, fruit $(5)$, tubers $(6)$, roots $(7,8)$, bark $(9,10)$, and rhizomes (11) of several plants.

Stinging nettle (Urtica dioica) accumulates a lectin of very low molecular weight, Urtica dioica agglutinin (UDA, ${ }^{3} M_{\mathrm{r}} 8000-$

\footnotetext{
${ }^{1}$ This research was supported by National Institute of Health Grant GM 29470.

${ }^{2}$ To whom correspondence should be addressed.

${ }^{3}$ Abbreviations used: UDA, Urtica dioica agglutinin; WGA, Wheat germ agglutinin; BSA, bovine serum albumin; Fuc, fucose; GlcNAc, $N$-acetylglucosamine; CETE, carboxyethylthioethyl; D.S., degree of substitution.
}

9000 ), solely in its rhizome, a specialized stem lying below ground (12). UDA consists of a single polypeptide chain and is a very stable protein. It agglutinates erythrocytes nonspecifically irrespective of blood group type and also induces the production of $\gamma$ interferon in human lymphocytes. We report herein the detailed carbohydrate binding properties of this lectin.

\section{MATERIALS AND METHODS}

Purification of Urtica dioica agglutinin. UDA was purified from the $0.1 \mathrm{~N} \mathrm{HCl}$ extract of rhizomes by affinity chromatography on chitin followed by SP-Sephadex column chromatography as previously reported (12).

Sugars and derivatives. $p$-Nitrophenyl- $N$-acetyl- $\beta$ lactosaminide was synthesized by N. Plessas of this laboratory. Synthetic oligosaccharides Gal $(\beta 1$ 4)GlcNAc( $\beta 1-6) \mathrm{Man}$, penta-2,6, and hepta (structures are shown in Table I) were provided by Dr. J. Arnarp and Dr. J. Lonngren of the University of Sweden. $N$ Glycolylneuraminic acid was purchased from Sigma Chemical Company (St. Louis, Mo.). N-Acetylneuraminic acid and its methyl ester were provided by Dr. G. W. Jourdian of this university and Dr. M. Flashner of Syracuse University, respectively. $N$-Acetylneu- 


\section{TABLE I}

Inhibition of UDA- $p$-AZophenYl- $\beta$-Chitobioside-BSA Precipitation by Low Molecular Weight Sugars

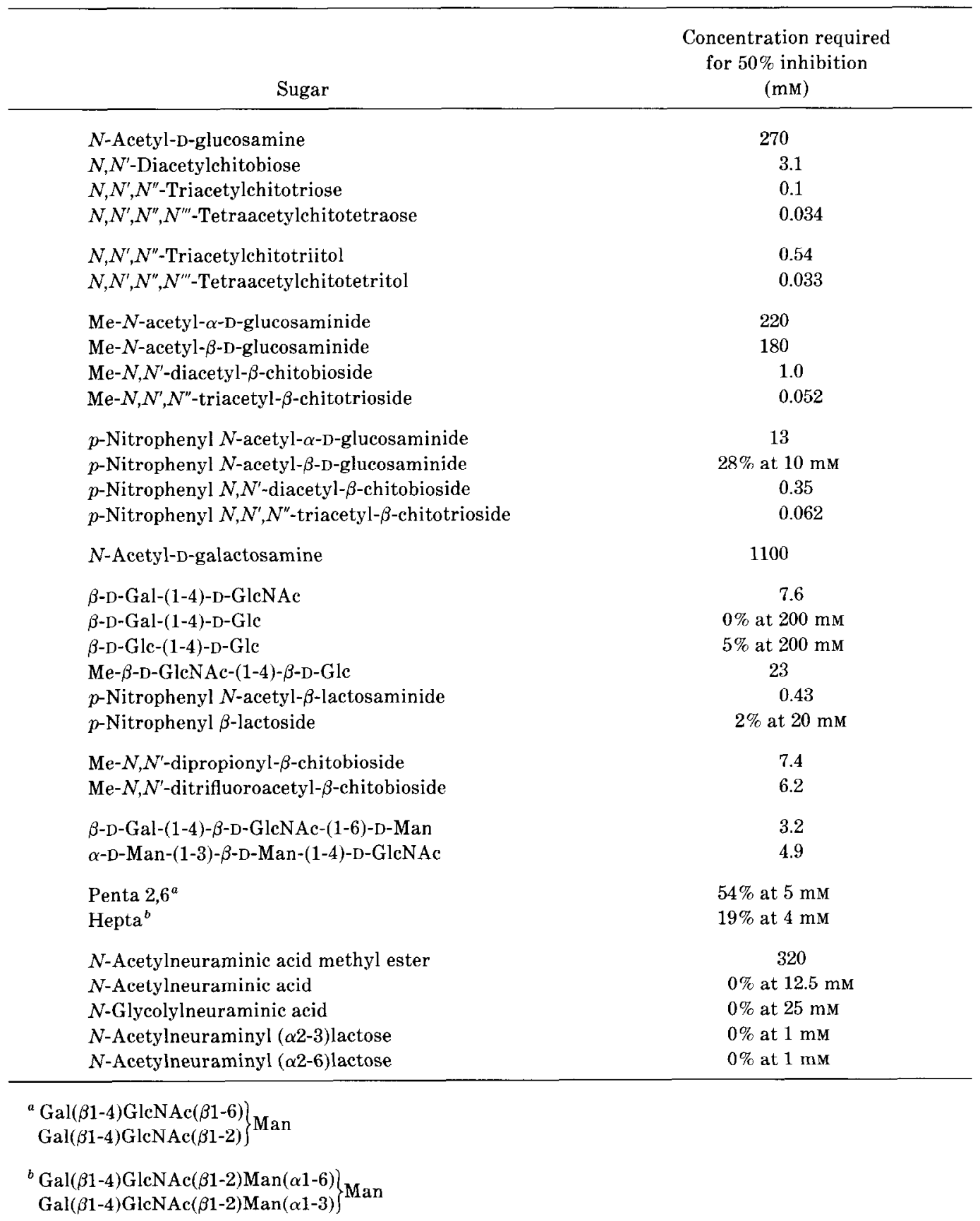

raminyl ( $\alpha 2-3)$ - and ( $\alpha 2-6)$-lactose were the gift of Dr. V. Ginsburg of National Institutes of Health. Other sugars were available from previous studies by this laboratory (13).

Glycoproteins and polysaccharides. Fetuin was purchased from Grand Island Biological Company (New
York). Gal( $\beta 1-4) G l c N A c-c a r b o x y e t h y l t h i o e t h y l$ (CETE) bovine serum albumin (BSA) [degree of substitution (D.S.) $=16 \mathrm{~mol} / \mathrm{mol})$ and GlcNAc( $\beta 1$ 4) $[$ Fuc $(\alpha 1-6)]$ GlcNAc-CETE-BSA (D.S. $=25-35 \mathrm{~mol} /$ mol) were purchased from the Swedish Sugar Company, Ltd. (Arlov, Sweden). Laminin was prepared by 
R. Knibbs of this laboratory from a murine tumor (EHS sarcoma) as previously reported (14). $p$-Azophenyl $\beta$-D-GlcNAc-BSA (D.S. $=13 \mathrm{~mol} / \mathrm{mol}$ ), $p$-azophenyl $\beta$-chitobioside-BSA (D.S. $=16 \mathrm{~mol} / \mathrm{mol}$ ), Gal( $\beta 1-4)$ GleNAc( $\beta 1-2)$ Man-BSA, asialofetuin, orosomucoid, asialoorosomucoid, keratan, and desulfated keratan were available from previous studies in this laboratory.

Precipitin and hapten inhibition reactions. Precipitin reactions were conducted by the modified method of So and Goldstein (15). Varying amounts of glycoconjugate were incubated with $26 \mu \mathrm{g}$ UDA in a total volume of $250 \mu \mathrm{l}$. After incubation for $1 \mathrm{~h}$ at $37^{\circ} \mathrm{C}$, the reaction mixture was kept at $4^{\circ} \mathrm{C}$ for $48 \mathrm{~h}$, centrifuged, and analyzed for protein in the precipitate. For inhibition experiments, varying amounts of sugars were added to the reaction mixture consisting of $26 \mu \mathrm{g}$ UDA and $40 \mu \mathrm{g} p$-azophenyl $N, N^{\prime}$-diacetyl- $\beta$-chitobioside$\mathrm{BSA}$ and reacted similarly. Since UDA showed a color intensity by Lowry's method higher than that of BSA due to its higher content of aromatic amino acid residues, the value of UDA obtained by this method was divided by a correction factor (1.55) derived from a comparison of the values obtained by Lowry's method with that from the Biuret method.

Equilibrium dialysis. The radioactive ligand was obtained by reduction of $N, N^{\prime}, N^{\prime \prime}, N^{\prime \prime \prime}$-tetraacetylchitotetraose with sodium borotritide. Equilibrium dialysis was performed in a multichambered dialysis cell (Terhnilah Instruments, New Jersey). One chamber contained $25 \mu \mathrm{M}$ UDA; the other contained ligand (1-1000 $\mu \mathrm{M})$ in $350 \mu$ l. Dialysis cells were rotated for $5-7$ days to attain equilibration. Aliquots $(10-50 \mu 1)$ were used for liquid scintillation counting and another $100 \mu \mathrm{l}$ was used for the determination of protein. Because of its small size, about $10 \%$ of UDA penetrated the membrane of the dialysis cell during the equilibration necessitating a correction, carried out as follows.

A preliminary Scatchard plot was made on the assumption that all of the UDA remained on one side of the dialysis membrane. The concentration of bound sugar was equated to the difference between concentrations of the sugar on both sides of the membrane. The ratio of the amount of bound sugar and the amount of protein $(r)$ was equated to the corresponding concentration ratio. Corrections for volume changes during equilibration were negligible. An approximate $K_{\mathrm{a}}$ for the high-affinity site was determined from a Scatchard plot. This $K_{\mathrm{a}}$ was used to correct for the small degree of UDA penetration into the sugar chamber. Because some of the sugar (concentration $[\mathrm{S}]_{\mathrm{b}}^{\mathrm{s}}$ ) in the sugar chamber is bound to the UDA in this chamber, the concentration of free sugar $[S]_{f}^{5}$ is less than the total concentration of sugar [S $\mathrm{S}_{0}^{\mathrm{s}}$ in the sugar chamber. The concentration of [S] was obtained by solving the equilibrium expression for [S] $[\mathrm{f}$ by using the approximate ratio of $K_{\mathrm{u}}$

$$
K_{\mathrm{a}}=\frac{[\mathrm{S}]_{\mathrm{b}}^{\mathrm{s}}}{\left(n[\mathrm{P}]_{0}^{\mathrm{s}}-[\mathrm{S}]_{\mathrm{b}}^{\mathrm{s}}\right)\left[\mathrm{S}_{\mathrm{f}}^{\mathrm{s}}\right.}=\frac{\left([\mathrm{S}]_{0}^{\mathrm{s}}-[\mathrm{S}]_{\mathrm{g}}^{\mathrm{s}}\right)}{\left(n[\mathrm{P}]_{0}^{\mathrm{s}}-[\mathrm{S}]_{0}^{\mathrm{s}}+[\mathrm{S}]_{\mathrm{f}}^{\mathrm{s}}\right)\left[\mathrm{S} \mathrm{f}_{\mathrm{f}}^{\mathrm{s}}\right.}
$$

where $[\mathrm{P}]_{0}^{8}$ represents the measured concentration of lectin in the sugar chamber and $n$, the total number of high affinity sites, is assumed for purposes of this correction to be unity. (Correction due to the binding of sugar to the low-affinity site of the UDA which penetrated into the sugar chamber was negligible). The ratio of the amount of bound sugar to the total amount of lectin in the lectin chamber $(r)$ was calculated by dividing the difference between the total sugar concentration in lectin side and $[\mathrm{S}]_{\mathrm{f}}^{\Omega}$ by the concentration of lectin in the lectin chamber as measured at equilibrium. The corrected values of $r$ and [S] were used to generate a second Scatchard plot which yielded a corrected value of $K_{\mathrm{a}}$. When this corrected value of $K_{\mathrm{a}}$ was used to correct again the data as outlined above, the resulting corrections were too small to produce a significant alteration in the Scatchard plot.

Binding parameters were obtained from nonlinear square fits to Eq. [2] by using a computer program (BMDX 85) from the Health Sciences Laboratory of the University of California, Los Angeles. The program obtains a least squares fit to functions by means of stepwise Gauss-Newton iterations of the parameters. The molecular weight of UDA was assumed to be 8500 for all the calculations (12).

Ultraviolet difference spectroscopy. UV-difference spectroscopic analysis was performed by using a Carry 219 double beam spectrophotometer at room temperature $\left(25^{\circ} \mathrm{C}\right)$. Yankeelov cells were used for recording the spectra but conventional cuvettes were also employed for the titration of UDA by $N$-acetylated chitooligosaccharides. For the titrations, aliquots of sugar solution (2.5-45 $\mathrm{mm}$ ) were added stepwise to the UDA solution $(20-40 \mu \mathrm{M})$, while the same amount of phosphate-buffered saline was added to the reference cell containing an identical UDA solution. The difference of the positive peak at $292 \mathrm{~nm}$ and the negative peak at $288 \mathrm{~nm}$ was measured and used as the index of hapten binding. Necessary corrections were made for the effect of dilution by the addition of sugar solution.

\section{RESULTS}

\section{Precipitation of Glycoconjugates by UDA}

The ability of several natural and synthetic glycoconjugates to precipitate with UDA was examined by the quantitative precipitation reaction (Fig. 1). UDA precipitated $p$-azophenyl $N, N^{\prime}$-diacetyl- $\beta$-chitobioside-BSA but did not precipitate a corresponding monosaccharide derivative, $p$-azophenyl $N$-acetyl- $\beta$-D-glucosaminideBSA. UDA also precipitated GlcNAc $(\beta 1-$ 


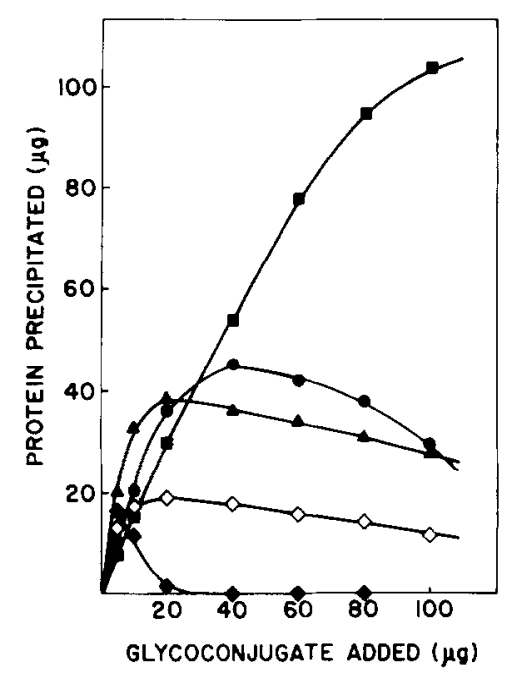

FIG. 1. Quantitative precipitation of glycoconjugates by UDA. -, $p$-Azophenyl- $\beta$-chitobioside-BSA; $\bullet$, keratan sulfate;, desulfated keratan; $\square$, laminin; $\boldsymbol{\Lambda}$, GlcNAc $(\beta 1-4)[\mathrm{Fuc}(\alpha 1-6)] \mathrm{GlcNAc-CETE-BSA}$.

4)[Fuc $(\alpha 1-6)]$ GlcNAc-CETE-BSA, which has a fucosylated $N, N^{\prime}$-diacetylchitobiose structure similar to the core region of many $N$-asparagine-linked glycoproteins. Both keratan sulfate and desulfated keratan, which contain the repeating -3$) \operatorname{Gal}(\beta 1$ 4)GlcNAc( $\beta 1$ - disaccharide residue, were precipitated by UDA; however, the desulfated species showed more extensive precipitation over a wider concentration range than the parent polysaccharide. Laminin also precipitated strongly with the lectin, whereas fetuin, orosomucoid, and their asialo-derivatives did not form a precipitate with UDA over the concentration range tested. Synthetic glycoconjugates containing the $N$-acetyllactosamine unit $[\mathrm{Gal}(\beta 1-4) \mathrm{GlcNAc}]$, i.e., Gal $(\beta 1-4) \mathrm{GlcNAc}-$ CETE-BSA and Gal( $\beta 1-4)$ GleNAc( $\beta 1-2)-$ Man-BSA, were not precipitated by the lectin.

\section{Inhibition of Precipitin Reaction by Haptenic Sugars}

To characterize the carbohydrate binding specificity of UDA, hapten inhibition experiments were performed with $p$-azophenyl $\beta$-chitobioside-BSA as the precipitating glycoconjugate. The concentration of sugar required for $50 \%$ inhibition as obtained from the inhibition curve for each sugar (Fig. 2) is listed in Table I.

Examination of the data in Table I reveals that for $\mathrm{N}$-acetylated chitooligosaccharides which were reported to be good inhibitors in a preliminary communication (12), there is a 90 -fold increase in inhibitory potency on going from GlcNAc to $(\mathrm{GlcNAc})_{2}$, and a further 30 -fold increase in inhibitory potency on going from $(\mathrm{GlcNAc})_{2}$ to (GleNAc) $)_{3}$. A much more modest 3-fold increase in inhibitory potency was seen on going from (GlcNAc) $)_{3}$ to $(\mathrm{GlcNAc})_{4}$. This 3 -fold increase in inhibitory potency associated with addition of a GlcNAc group to $(\mathrm{GlcNAc})_{3}$ was found to be comparable to that seen upon formation of the $\beta$-methylglycoside of $(\mathrm{GlcNAc})_{3}$. These results suggest that the carbohydrate binding site of UDA is complementary to an $N, N^{\prime}, N^{\prime \prime}$-triacetylchitotriose unit, and similar to that of the lectins from wheat germ (WGA) (16) and Datura stramonium seeds (13).

Reduction of $N, N^{\prime}, N^{\prime \prime}$-triacetylchitotriose to the corresponding alditol decreased its inhibitory power fivefold, making it similar in potency to that of the methylglycoside of the disaccharide. This result suggests that the open-chain alditol portion of the reduced trisaccharide does not bind to this lectin as well as the intact sugar residue. On the other hand, reduction of $N, N^{\prime}, N^{\prime \prime}, N^{\prime \prime \prime}$-tetraacetylchitotetraose did not significantly change its affinity in comparison to the parent sugar, supporting the above conclusion regarding the size of the carbohydratc binding sitc.

Introduction of the $p$-nitrophenyl aglycon in the $\beta$-anomeric configuration markedly enhanced the inhibitory power (20-fold) of $N$-acetyl-D-glucosamine as compared to the parent sugar. Similar enhancement was also observed with $N$ acetyllactosamine, but not with the noninhibitory disaccharide lactose, suggesting that this phenomenon is dependent upon the specific binding of sugars to the lectin. This enhancement in binding produced by the $p$-nitrophenyl aglycon decreased with an increase in the number of sugar residues; that is, the $p$-nitrophenylglycoside of 


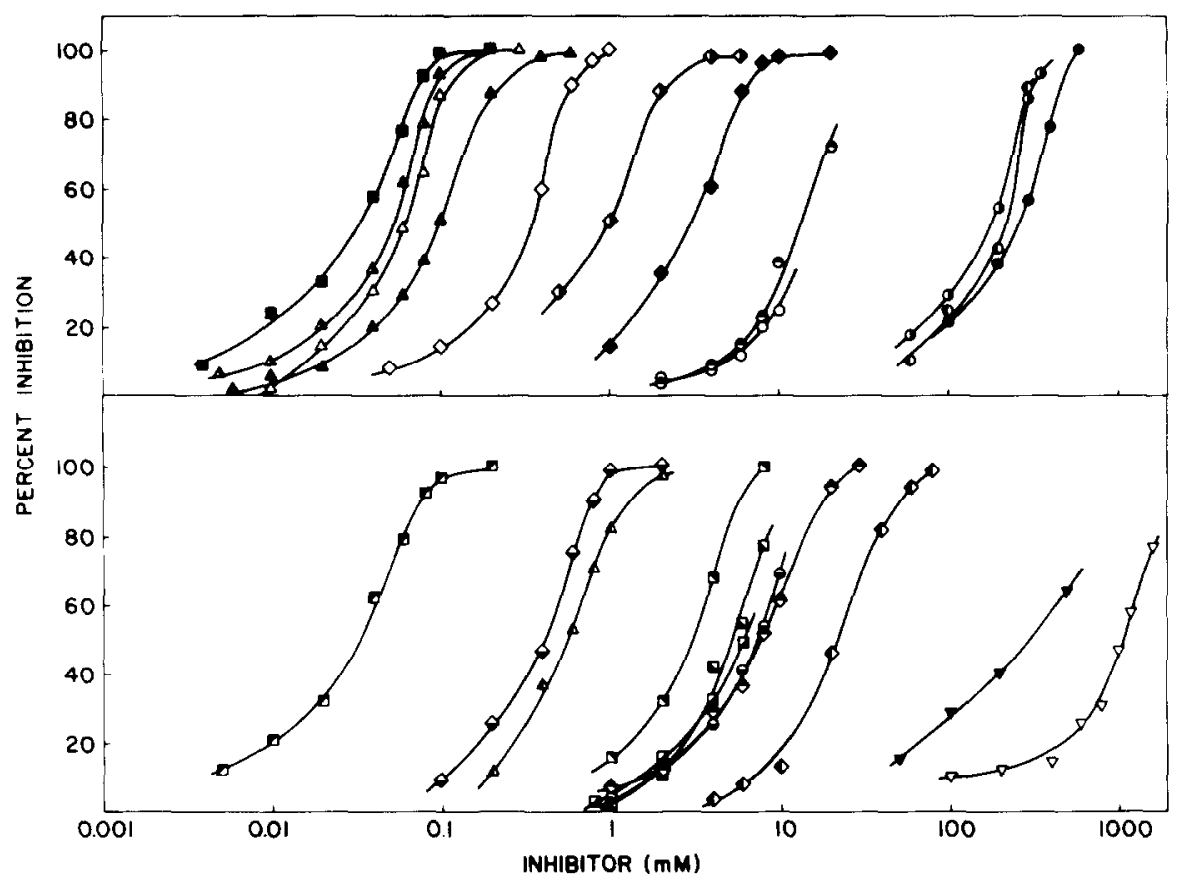

FIG. 2. Inhibition of UDA- $p$-Azophenyl- $\beta$-chitobioside-BSA precipitation by low molecular weight

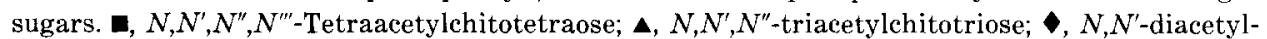
chitobiose; $\bullet$-acetyl-D-glucosamine; $\boldsymbol{0}$, methyl $N$-acetyl- $\beta$-D-glucosaminide; $\boldsymbol{0}$, methyl $N$-acetyl$\alpha$-D-glucosaminide; $\triangleleft$, methyl $N, N^{\prime}$-diacetyl- $\beta$-chitobioside; $\boldsymbol{\Delta}$, methyl $N, N^{\prime}, N^{\prime \prime}$-triacetyl- $\beta$-chitotrioside; $\bigcirc, p$-nitrophenyl $N$-acetyl- $\beta$-D-glucosaminide; $\sigma, p$-nitrophenyl $N$-acetyl- $\alpha$-D-glucosaminide; $\vartheta$,nitrophenyl $N, N^{\prime}$-diacetyl- $\beta$-chitobioside; $\Delta, p$ nitrophenyl $N, N^{\prime}, N^{\prime \prime}$-triacetyl- $\beta$-chitotrioside;

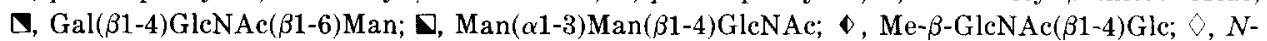
acetyllactosamine; $\boldsymbol{\square}, N, N^{\prime}, N^{\prime \prime}, N^{\prime \prime \prime}$-tetraacetylchitotetritol; $0, p$-nitrophenyl $N$-acetyl- $\beta$-lactosaminide; $\triangle, N, N^{\prime \prime}, N^{\prime \prime}$-triacetylchitotriitol; $\square$, methyl $\beta$ - $N, N^{\prime}$-ditrifluoroacetylchitobioside; $\ominus$, methyl $\beta$-dipropionylchitobioside; $\nabla, N$-acetyl-D-galactosamine; $\boldsymbol{\nabla}$, methyl ester of $N$-acetylneuraminic acid.

the disaccharide was nine times more inhibitory than the parent sugar, whereas almost no increase in affinity was observed with the trisaccharide.

Substitution of the acetyl groups in methyl $N, N^{\prime}$-diacetyl- $\beta$-chitobioside with propionyl or trifluoroacetyl groups decreased the inhibitory power six- to sevenfold, suggesting steric hindrance by the bulkier propionyl groups and possibly an unfavorable polar or electronic effect produced by the polar, highly electronegative trifluoroacetyl groups. Chitobiose itself was a very poor inhibitor, probably because of an unfavorable electrostatic interaction of its $\mathrm{NH}_{3}^{+}$groups with the lectin molecule. Interestingly, $N$-acetyllactosamine, Gal$(\beta 1-4)$ GlcNAc, which was reported to be a good inhibitor of the $D$. stramonium lectin
(13) but not of WGA (17) or potato lectin (6), was a good inhibitor of UDA, being approximately one-half as potent as $N, N^{\prime}$-diacetylchitobiose. On the other hand, both Gal( $\beta 1-4)$ Glc and Glc $(\beta 1-4) \mathrm{Glc}$, the glucose analogs of $N$-acetyllactosamine and $N, N^{\prime}$ diacetylchitobiose, were not inhibitory. Surprisingly, the $\beta$-methyl glycoside of GlcNAc $(\beta 1-4)$ Gle was more than 20 times less inhibitory than the corresponding methyl $\beta$-glycoside of $\operatorname{GlcNAc}(\beta 1-4) \mathrm{Glc}$ NAc. These observations are discussed below in terms of the subsite specificity of this lectin.

Two trisaceharides, Gal $(\beta 1-4) \mathrm{GlcNAc}-$ $(\beta 1-6)$ Man and $\operatorname{Man}(\alpha 1-3) \operatorname{Man}(\beta 1-4) G l c-$ NAc, both showed an inhibitory power similar to that of $N, N^{\prime}$-diacetylchitobiose. These observations indicate that the ad- 
dition of a hexose unit to the reducing or nonreducing termini of a $\mathrm{Gal}(\beta 1-4) \mathrm{GlcNAc}$ or Man( $\beta 1-4) G l c N A c$ unit does not impair their binding to UDA.

Synthetic oligosaccharides which have branched structures analogous to those of the complex-type carbohydrate chains of glycoproteins, and which were reported to have a much greater affinity to $D$. stramonium lectin as compared to $N$-acetyllactosamine (13), did not show an enhanced affinity for UDA. The extremely high affinity of the penta 2,6 biantennary pentasaccharide for the $D$. stramonium lectin (penta 2,6 was 500 times more inhibitory than $N$-acetyllactosamine) was interpreted as indicating the possible simultaneous binding of two Gal $(\beta 1-4)$ GlcNAc units of these oligosaccharides to the two binding sites of this lectin. The much lower affinity of the same oligosaccharides for UDA, suggests that these branched oligosaccharides cannot bind to UDA in a similar fashion.

Interestingly, the methyl ester of $N$-acetylneuraminic acid weakly inhibits the precipitation reaction with UDA to almost the same extent as $N$-acetylglucosamine. Among plant lectins, only WGA has been reported to show a similar inhibition by sialic acid derivatives $(18,19)$. The precipitation of elder bark lectin (10) with glycoconjugates, however, is also inhibited by low concentrations (less than $0.1 \mathrm{~mm}$ ) of sialic acid-containing oligosaccharides such as sialyllactose but not by sialic acid itself (N. Shibuya et al., unpublished results). Sialyllactose at concentrations of 1 $\mathrm{mM}$, however, does not inhibit precipitation of glycoconjugates by UDA.

\section{Equilibrium Dialysis}

The Scatchard plot of the results of equilibrium dialysis with $\left[{ }^{3} \mathrm{H}\right] N, N^{\prime}, N^{\prime \prime}, N^{\prime \prime \prime}-$ tetraacetylchitotetritol showed marked curvature (Fig. 3), which indicated the presence of negative cooperativity between the binding sites or the presence of highand low-affinity binding sites in this lectin (20). The Scatchard plots for such a system

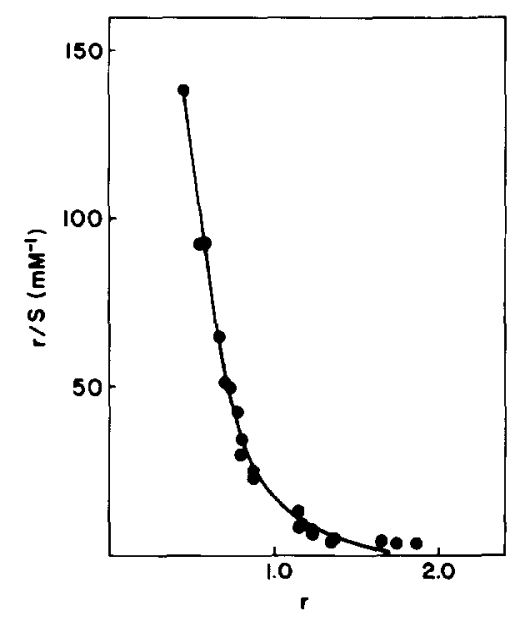

FIG. 3. Binding of $\left[{ }^{3} \mathrm{H}\right] N, N^{\prime}, N^{\prime \prime}, N^{\prime \prime \prime}$-tetraacetylchitotetritol to UDA. Results obtained by equilibrium dialysis were plotted according to Scatchard, after correction, as described under Materials and Methods.

can be conveniently interpreted in lerms of Eq. [2], ${ }^{4}$

$$
\begin{array}{r}
\frac{r}{[\mathrm{~S}]}=\frac{n_{1}}{[\mathrm{~S}]+G_{1}}+\frac{n_{2}}{[S]+G_{2}}+\frac{n_{3}}{[\mathrm{~S}]+G_{3}} \\
+\cdots+\frac{n_{\mathrm{i}}}{[\mathrm{S}]+G_{\mathrm{i}}}
\end{array}
$$

where $n_{\mathrm{i}}$ denotes the number of sites having a dissociation constant $G_{\mathrm{i}},[\mathrm{S}]$ is the concentration of free ligand, and $r$ is the ratio of bound ligand and lectin. A two-site model $\left(n_{\mathrm{i}}=0\right.$ when $\left.i>2\right)$ fit the data well and a computer nonlinear least squares fit of the data to Eq. [2] yielded the values $n_{1}$ $=0.73 \pm 0.04, G_{1}=0.24 \times 10^{-5} \mathrm{M}, n_{2}=1.04$ $\pm 0.28, G_{2}=0.15 \times 10^{-3} \mathrm{M}$.

It is important to note that Eq. [2] is a mathematical transformation which facilitates data analysis, and which for the purpose of convenient data analysis represents the equilibrium system as a mixture of independent macromolecular binding sites. If the binding sites on the macromolecule were truly independent, $G_{1}$ and

\footnotetext{
${ }^{4}$ It should be noted that Eq. [2] will not fit the data for a system which exhibits positive cooperativity and still yield real values for the parameters $G_{i}$.
} 
$G_{2}$ would be numerically equivalent to the values of microscopic dissociation constants for the high- and low-affinity sites. It has been shown by Simms (21) that in cases where $n_{1}=n_{2}=1$ the macroscopic equilibrium constants for association $\left(K_{\mathrm{a} 1}\right.$ and $K_{\mathrm{a} 2}$ ) as defined by

$$
\begin{aligned}
& \mathrm{S} \quad \mathrm{S} \\
& +K_{\mathrm{a} 2}+K_{\mathrm{k} 2} \\
& \mathrm{P} \leftrightharpoons \mathrm{PS} \leftrightharpoons \mathrm{PS}_{2}
\end{aligned}
$$

are related to $G_{1}$ and $G_{2}$ by Eqs. [4] and [5] regardless of whether the sites are independent.

$$
\begin{aligned}
K_{\mathrm{a} 1} & =G_{1}^{-1}+G_{2}^{-1} \\
K_{\mathrm{a} 2}^{-1} & =G_{1}+G_{2}
\end{aligned}
$$

Thus, $K_{\mathrm{a} 1}=4.2 \times 10^{5} \mathrm{M}^{-1}$ and $K_{\mathrm{a} 2}=6.6$ $\times 10^{3} \mathrm{M}^{-1}$.

\section{Ultraviolet Difference Spectroscopic \\ Analysis of UDA-Ligand Interaction}

Binding of chitooligosaccharides to UDA induced a uv difference spectrum with peaks at 292,285 , and $275 \mathrm{~nm}$ (Fig. 4), which suggests perturbation of tryptophanyl residues in UDA (22). When $p$-nitrophenylglycosides of (GlcNAc) $)_{2}$ and (GleNAc) $)_{3}$ were used as ligands, an additional nega-

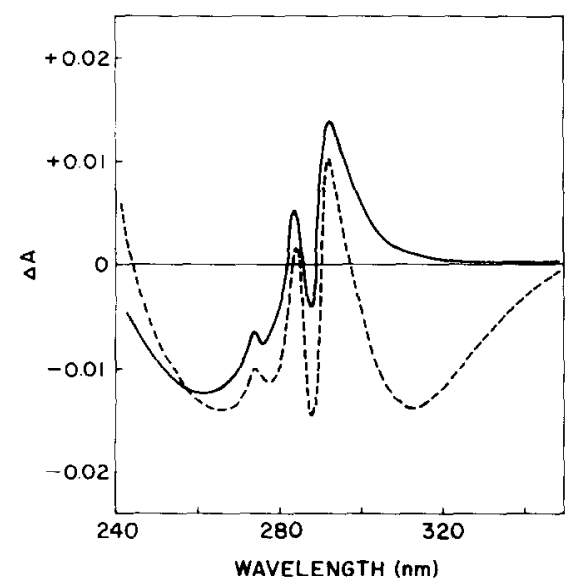

FlG. 4. Ultraviolet difference spectrum of UDA induced by chitooligosaccharides. Spectrum was recorded in the presence of $100 \mu \mathrm{M} N, N^{\prime}, N^{\prime \prime}$-triacetylchitotriose (solid line) or $100 \mu \mathrm{M} p$-nitrophenyl $N, N^{\prime}, N^{\prime \prime}$-triacetyl- $\beta$-chitotrioside (broken line).

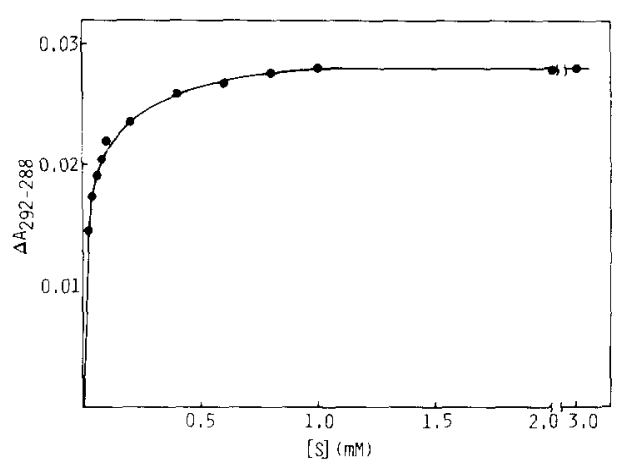

FIG. 5. Titration curve for $N, N^{\prime}, N^{\prime \prime}, N^{\prime \prime \prime}$-tetraacetylchitotetraose.

tive peak appeared with a broad maximum at $312 \mathrm{~nm}$, indicating the perturbation of the $p$-nitrophenyl group in the ligand (23).

The dependence of the change in absorbance $(\Delta A)$ associated with ligand binding on the total concentration of chitotetraose $\left[\mathrm{S}_{\mathrm{t}}\right]$ depicted in Fig. 5 and Table II suggests that UDA contains nonequivalent binding sites. If UDA contained one or two equivalent binding sites, the concentration of bound $\left[S_{b}\right]$ and free $[S]$ ligand could be obtained from the expressions

TABLE II

DEPENDENCE of THE APPARENT Equilibrium Constant For the Binding of $N, N^{\prime}, N^{\prime \prime}, N^{\prime \prime \prime}-$

TÉTRACETYLCHITOTETROSE TO UDA AS DETERMINED BY SPECTROPHOTOMETRIC TITRATION ${ }^{a}$

\begin{tabular}{cccc}
\hline & & \multicolumn{2}{c}{$10^{5} K\left(\mathrm{M}^{-1}\right)$} \\
\cline { 3 - 3 }$(\mathrm{S} \mathrm{t}]$ & $10^{3} \Delta A$ & $n=1$ & $n=2$ \\
\hline 0.020 & 14.6 & 1.14 & -12.7 \\
0.040 & 17.4 & 0.60 & 1.08 \\
0.060 & 19.1 & 0.46 & 0.66 \\
0.080 & 20.5 & 0.41 & 0.54 \\
0.100 & 22.0 & 0.42 & 0.53 \\
0.200 & 23.6 & 0.30 & 0.32 \\
0.40 & 25.9 & 0.33 & 0.34 \\
1.00 & 28.0 & & \\
3.00 & 28.0 & & \\
\hline
\end{tabular}

${ }^{a}$ The conditions for the titration are described under Materials and Methods. Values of $K$ were calculated from Eqs. [6]-[8] with $\Delta A_{\max }$ set at 0.0280 and a lectin concentration $\left[\mathrm{P}_{\mathrm{t}}\right]$ of $20 \mu \mathrm{M}$. 


$$
\begin{aligned}
{\left[\mathrm{S}_{\mathrm{b}}\right] } & =n\left[\mathrm{P}_{\mathrm{t}}\right] \Delta A / \Delta A_{\max } \\
{[\mathrm{S}] } & =\left[\mathrm{S}_{\mathrm{t}}\right]-\left[\mathrm{S}_{\mathrm{b}}\right]
\end{aligned}
$$

when lectin at a concentration $\left[\mathrm{P}_{\mathrm{t}}\right]$ is $\mathrm{ti}$ trated with increasing concentrations $\left[\mathrm{S}_{\mathrm{t}}\right]$ of carbohydrate ligand. In Eq. [6], $\Delta A / A_{\max }$ is the ratio of the magnitude of the absorbance difference at a ligand concentration of $\left[\mathrm{S}_{t}\right]$ and the limiting value of the absorbance difference. The data in Table II show that the apparent value of the equilibrium constant $(K)$ for association as calculated from the relationship

$$
K=\frac{\left[\mathrm{S}_{\mathrm{b}}\right]}{\left(n\left[\mathrm{P}_{\mathrm{t}}\right]-\left[\mathrm{S}_{\mathrm{b}}\right]\right)[\mathrm{S}]}
$$

varies with the ligand concentration regardless of whether we assume a value of 1 or 2 for $n$, the number of sugar binding sites in UDA. Similar results were obtained upon analysis of the spectrophotometric titrations for several other ligands, including $N, N^{\prime}$-diacetylchitobiose and $N, N^{\prime}, N^{\prime \prime}$-triacetylchitotriose.

These observations are diagnostic of heterogeneity with respect to the binding sites, and are consistent with the finding of nonequivalent carbohydrate binding sites in our studies of the binding of $\left[{ }^{3} \mathrm{H}\right] N, N^{\prime}, N^{\prime \prime}, N^{\prime \prime \prime}$-tetraacetylchitotetritol to UDA by equilibrium dialysis. Quantitative analysis of the dependencies of $\Delta A$ on ligand concentration in spectrophotometric titrations of UDA is complicated by the possibility that the molar absorptivity change for binding of ligand to one site of UDA is different from that for binding of ligand to the other site. In light of this problem, equilibrium dialysis appears preferable to spectrophotometric titration as a method for evaluation of equilibrium constants for formation of complexes between carbohydrate ligands and polyvalent lectins with nonequivalent binding sites. With regard to analysis of spectrophotometric titration data in Table II, it is interesting to note that a plot of $\left[\mathrm{S}_{t}\right] / \Delta A$ vs $\left[\mathrm{S}_{\mathrm{t}}\right]$ is linear (Fig. 6). A plot of $\left[\mathrm{S}_{\mathrm{t}}\right] / \Delta A$ vs $\left[\mathrm{S}_{\mathrm{t}}\right]$ is often used to determine the equilibrium constant for association of a ligand

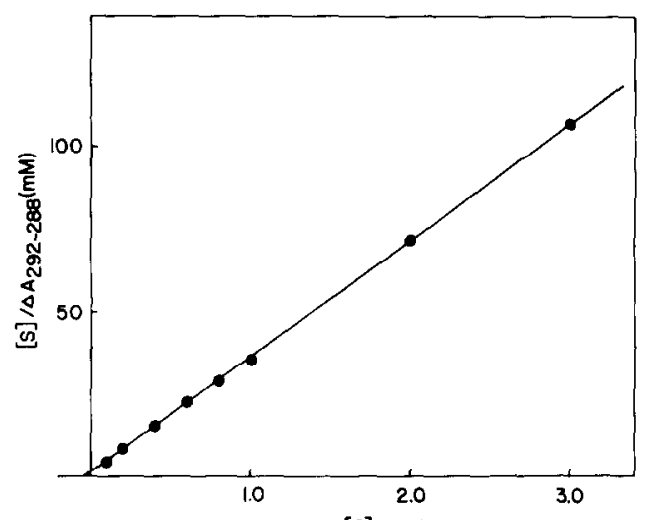

[s] $(\mathrm{mM})$

Fig. 6. Dependence of $\left[S_{t}\right] / \Delta A$ on $\left[S_{t}\right\rfloor$ for $N, N^{\prime}, N^{\prime \prime}, N^{\prime \prime \prime}-$ tetraacetylchitotetraose. Both $\left[\mathrm{S}_{\mathrm{t}}\right] / \Delta A$ and $\left[\mathrm{S}_{\mathrm{t}}\right]$ were obtained from the same experiment used for Fig. 5 .

with a protein (24), since it follows from Eq. [8] that if the sites are equivalent

$$
\frac{1}{K}=\left(\frac{\Delta A_{\max }}{\Delta A}-1\right)[\mathrm{S}]
$$

and

$$
\frac{[\mathrm{S}]}{\Delta A}=\frac{1}{K \Delta A_{\max }}+\frac{[\mathrm{S}]}{\Delta A_{\max }}
$$

In cases where $\left[S_{t}\right] \gg\left[P_{t}\right]$, it can be assumed that $\left[\mathrm{S}_{\mathrm{t}}\right] \approx[\mathrm{S}]$, and a plot of $\left[\mathrm{S}_{\mathrm{t}}\right]$ $\Delta A$ vs $\left[\mathrm{S}_{\mathrm{t}}\right]$ would be expected to be linear when the binding sites are equivalent and noninteracting. The assumption that $\left[\mathrm{S}_{\mathrm{t}}\right]$ $\approx[\mathrm{S}]$ does not hold for the data in Table II, however, since $\left[S_{t}\right]$ is comparable to $\left[P_{t}\right]$ and significantly larger than [S] for several members of the data set. The observation of a linear dependence of $\left[\mathrm{S}_{\mathrm{t}}\right] / \Delta A$ on $\left[\mathrm{S}_{\mathrm{t}}\right]$ illustrates the possibility of obtaining artifactually linear plots of $\left[\mathrm{S}_{\mathrm{t}}\right] / \Delta A$ vs $\left[\mathrm{S}_{\mathrm{t}}\right]$ (or linear plots of $1 / \Delta A$ vs $1 /\left[\mathrm{S}_{\mathrm{t}}\right]$ ) and erroneous values for binding constants under conditions where the assumption $\left[\mathrm{S}_{\mathrm{t}}\right] \approx[\mathrm{S}]$ is not justified.

\section{DISCUSSION}

The results obtained in this study indicate that UDA has an extended carbohydrate binding site complementary to an $N, N^{\prime}, N^{\prime \prime}$-triacetylchitotriose unit and similar to that of WGA (16) and the D. stramonium lectin (13). The structural feature 
common to all oligosaccharides with which UDA (as well as all chitodextrin-binding lectins) interacts is the presence of one or more $\mathrm{N}$-acetyl-D-glucosamine units bearing a glycosyl residue at the $\mathrm{C}-4$ position. The 4-O-substituted GlcNAc group may be situated at the reducing or at the internal position of an oligosaccharide. Oligosaccharides containing a single $N$-acetyl-Dglucosamine unit at the nonreducing end bind poorly to the lectin. Thus, the disaccharides $N, N^{\prime}$-diacetylchitobiose and Gal $(\beta 1-4)$ GlcNAc and the trisaccharides Gal $(\beta 1-4)$ GlcNAc $(\beta 1-6)$ Man and $\operatorname{Man}(\alpha 1-$ 3)Man( $(\beta 1-4)$ GlcNAc all exhibit comparable affinities for UDA as reflected by their similar inhibitory potencies ( 3 to $7 \mathrm{~mm}$ for $50 \%$ inhibition), whereas the disaccharide glycoside methyl $\beta$-GleNAc $(\beta 1-4) \mathrm{Glc}$ interacts weakly with the lectin $(23 \mathrm{~mm}$ for $50 \%$ inhibition). These data together with the observation that $N, N^{\prime}, N^{\prime \prime}$-triacetylchitotriose is a 30 -fold more potent inhibitor of UDA than $N, N^{\prime}$-diacetylchitobiose suggest that the lectin contains three subsites. These are designated A, B, and C in Fig. 7, which illustrates possible binding modes for several oligosaccharides. More information is necessary to substantiate and

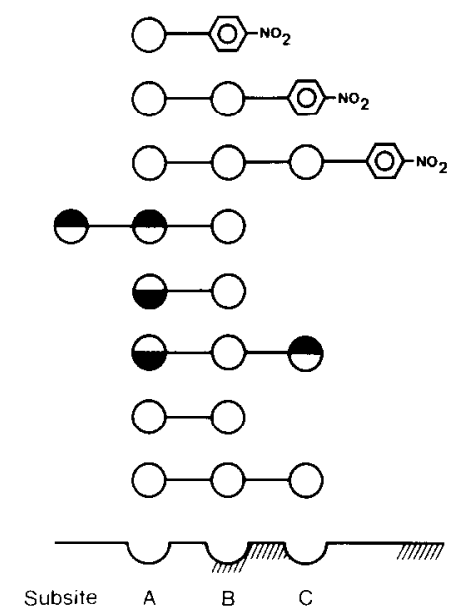

FIG. 7. A model of the carbohydrate binding site of UDA. $\bigcirc$, GlcNAc; $\bullet$, Man; $\ominus$, Gal. All the glycosidic linkages are $\beta 1-4$, except that between the two mannose residues $(\alpha 1-3)$ and $N$-acetylglycosamine and mannose $(\beta 1-6)$. Hydrophobic binding regions are depicted by $\|\mid\| \|$. characterize further this model for the UDA binding site, which is similar to that proposed for WGA $(27,28)$ and the potato (29) and D. stramonium lectins (13).

Hapten inhibition with $p$-nitrophenyl glycosides suggests the presence of a nonpolar region adjacent to the carbohydrate binding site of UDA, similar to that observed for several other plant lectins (13, $25,26)$. The observation that the enhancement in affinity produced by the $p$-nitrophenyl aglycon was most pronounced for the monosaccharide, decreased in the disaccharide, and virtually disappeared in the trisaccharide suggests that this hydrophobic interacting site is localized near subsite B or between subsites B and C (see Fig. 7). However, the results of the uv difference spectroscopic studies suggest that the environment of the $p$-nitrophenyl group of bound trisaccharide as well as bound disaccharide and monosaccharide is perturbed, indicating that the lectin interacts with the aglycon of all three bound ligands (23). The unequal effects of this interaction of the binding of these glycosides suggest the possibility that the nitrophenyl group in these ligands interacts with the lectin differently (perhaps with different apolar domains).

The binding modes of $N$-acetylneuraminic acid to UDA and WGA $(18,19)$ may be similar in that they depend on the similarity in configuration of the $\mathrm{C}-5(\mathrm{~N}$-acetamido group) and the C-4 (hydroxyl group) positions of $N$-acetylneuraminic acid with $N$-acetyl-D-glucosamine at positions C-2 ( $N$-acetamido group) and C-3 (hydroxyl group) of the pyranose ring [cf. Ref. (19)].

In almost all cases, linear Scatchard plots suggesting the presence of equivalent, noninteracting binding sites have been obtained for the binding of carbohydrate ligands to plant lectins. Thus, the finding of marked curvature in the Scatchard plot for UDA (Fig. 3) was surprising. To our knowledge, there has been only one previous report of a curvilinear Scatchard plot for the binding of carbohydrate (lactose) to a plant lectin (ricin) (30). Curvilinearity in a Scatchard plot is often interpreted as 
being indicative of the existence of negative cooperativity between two binding sites, or the presence of two binding sites with intrinsically different affinities. The presence of two equivalent binding sites on a single polypeptide chain would be expected to require the existence of two similar structural domains. It will be interesting to determine whether the structure of UDA is consistent with this notion.

Although the biological significance of the accumulation of this unique lectin in a plant rhizome remains to be established, the carbohydrate binding specificity of UDA toward $N$-acetylated chitooligosaccharides and other oligosaccharides as revealed in this work, together with UDA's marked stability (it is stable at $80^{\circ} \mathrm{C}, \mathrm{pH}$ 4.0, and also in $0.1 \mathrm{~N} \mathrm{HCl)} \mathrm{(12),} \mathrm{suggests}$ that IIDA will be useful as a tool for the detection, preparation, and isolation of cell surface glycoconjugates.

\section{REFERENCES}

1. Lis, H., AND Sharon, N. (1984) in Biology of Carbohydrates (Ginsburg, V., and Robbins, P., eds.), Vol. 2, pp. 1-85, Wiley, New York.

2. Goldstein, I. J., AND Hayes, C. E. (1978) $A d v$. Carbohydr. Chem. Biochem. 35, 127-340.

3. Talbot, C. F., And Etzler, M. E. (1978) Biochemistry 17, 14741479.

4. Lamb, J. E., Shibata, S., ANd Goldostein, I. J. (1983) Plant Physiol. 71, 879-887.

5. Kilpatrick, D. C. (1980) Biochem. J. 185, 269-272.

6. Allen, A. K., And Neuberger, A. (1973) Biochemistry 135, 307-314.

7. Dazzo, F. B., YANKE, W. E., AND BRILl, W. J. (1978) Biochim. Biophys. Acta 539, 276-286.

8. Peumans, W. J., Nsimba-Lubaki, M., Carlier, A. R., and Van Driessche, E. (1984) Planla 160, $222-228$.

9. Horejsi, V., Haskovec, C., AND Kocourek, J. (1978) Biochim. Biophys. Acta 532, 98-104.

10. Broekaert, W. F., Nsimba-Lubaki, M., Peeters,
B., And Peumans, W. J. (1984) Biochem. J. 221, 163-169.

11. Peumans, W. J., Nsimba-Lubaki, M., Peeters, B., and Broekaert, W. F. (1984) Planta 164, 7582.

12. Peumans, W. J., De Ley, M., And Broekaert, W. F. (1984) FEBS Lett. 177, 99-103.

13. Crowley, J. F., Goldstein, I. J., ARnarP, J., AND Lonngren, J. (1984) Arch. Biochem. Biophys. 231, 524-533.

14. Shibata, S., Peters, B., Roberts, D. D., GoldSTEIN, I. J., AND LIOTTA, L. A. (1982) FEBS Lett. 142, 194-198.

15. So, L. L., AND Goldstein, I. J. (1967) J. Biol. Chem. 242, 1617-1622.

16. Allen, A. K., Neuberger, A., AND Sharon, N. (1973) Biochem. J. 131, 155-162.

17. Goldstein, I. J., Hammarström, S., and SundBLAD, G. (1975) Biochim. Biophys. Acta 405, 5356.

18. Bhavanandan, V. P., and Katlic, A. W. (1979) J. Biol. Chem. 254, 4000-4008.

19. Peters, B. D., Ebisu, S., Goldstein, I. J., And Flashner, M. (1979) Biochemistry 18, 55055511.

20. Kabat, E. A. (1976) Structural Concepts in Immunology and Immunochemistry, pp. 95-118, Holt, Reinhart \& Winston, New York

21. Simms, H. S. (1926) J. Amer. Chem. Soc. 48, 12391250 .

22. Herskovits, T. T., and Sorensen, S. M. (1968) Biochemistry 7, 2523-2532.

23. Hassing, G. S., and Goldstein, I. J. (1970) Eur. J. Biochem. 16, 549-556.

24. Matsumoto, I., Jimbo, A., Kitagaki, H., GoLovtchenko-Matsumoto, A. M., and Seno, N. (1980) J. Biochem. 88, 10931096.

25. Poretz, R. D., AND Goldstein, I. J. (1971) Biochem. Pharmacol. 20, 2727-2739.

26. Murphy, L. A., AND Goldstein, I. J. (1979) Biochemistry 18, 4999-5005.

27. WRIGHT, C. S. (1980) J. Mol. Biol. 141, 267-291.

28. WRIGHT, C. S. (1984) J. Mol. Biol. 178, 91-104.

29. Jimbo, A., Seno, N., And Matsumoto, I. (1984) $J$. Biochem. 95, 267-275.

30. ZENTZ, C., FrÉnOY, J. P., AND BOURRILlon, R. (1978) Biochim. Biophys. Acta 536, 18-26. 